\title{
Final Report for "Toward quantifying kinetics of biotic and abiotic metal reduction with electrical geophysical methods" DE-FG02-08ER64520 \\ PIs: Kamini Singha and Susan Brantley
}

Summary: Although changes in bulk electrical conductivity in aquifers have been attributed to microbial activity, electrical conductivity has never been used to infer biogeochemical reaction rates quantitatively. To explore the use of electrical conductivity to measure reaction rates, we conducted iron oxide reduction experiments of increasing biological complexity. To quantify reaction rates, we proposed composite reactions that incorporated the stoichiometry of five different types of reactions: redox, acid-base, sorption, dissolution/precipitation, and biosynthesis. In batch and column experiments, such reaction stoichiometries inferred from a few chemical measurements allowed quantification of the Fe-oxide reduction rate based on changes in electrical conductivity. The relationship between electrical conductivity and fluid chemistry did not hold during the latter stages of the column experiment when electrical conductivity increased while fluid chemistry remained constant. Growth of an electrically conductive biofilm could explain this late stage electrical conductivity increase. The measured electrical conductivity increase is consistent with a model proposed by analogy from percolation theory that attributes the increased conductivity to growth of biofilms with conductivity of $\sim 5.5 \mathrm{~S} / \mathrm{m}$ in at least $3 \%$ of the column pore space. We followed up this work with reactive transport modeling to estimate the production of biomass, and linked that with a pore-scale electrical conductivity model to estimate changes in bulk electrical conductivity associated with a growing biomass.

This work demonstrates that measurements of electrical conductivity and flow rate, combined with a few direct chemical measurements, can be used to quantify biogeochemical reaction rates in controlled laboratory situations and may be able to detect the presence of biofilms. This approach may help in designing future field experiments to interpret biogeochemical reactivity from conductivity measurements.

\section{Overview of Project:}

The goal of this two-year Exploratory Grant was to develop conceptual and numerical models that could be used to relate changes in electrical geophysical parameters to rates of metal reduction, bacterial growth, mineral dissolution and precipitation. We collected biogeochemical and electrical data on well-constrained batchand column- scale laboratory setups of increasing complexity, and developed conceptual and numerical models for the interpretation of the geophysical data for our laboratory experiments. We proposed to identify contributions from changes in ionic strength, redox, biofilm formation, permeability, and precipitation from the electrical methods in conjunction with geochemical measurements to quantify the associated biogeochemical processes through time at the flask and column scale. These analyses improved quantification of real-time kinetics at the lab scale, laying the groundwork for how to best extrapolate to the field.

We conducted three sets of batch experiments with goethite as the electron acceptor. The simplest experiments (abiotic), designed so that reduction occurred without a biological catalyst, contained ascorbic acid as the electron donor. In vitro experiments, conducted to include a biogeochemical reaction without the complication of whole cells, 
contained Na-formate as the electron donor and total membrane fractions (TM) from Shewanella oneidensis MR-1, a known iron reducer. The abiotic, in vitro, and in vivo Fe reduction batch experiments were characterized by different rates of change of fluid electrical conductivity, $\mathrm{pH}$, and dissolved $\mathrm{Fe}(\mathrm{II})$ concentrations ([Fe(II)]) over hours. These data were interpreted using composite chemical reactions validated with chemical measurements. We found that surface area-normalized reduction rates of goethite increase from abiotic to in vivo to in vitro reduction for similar concentrations of electron donor and acceptor, and the electrical geophysical measurements were key to developing meaningful composite reactions. We developed an iterative method for predicting and testing composite chemical reactions that can be used in conjunction with changes in fluid electrical conductivity to measure the rate of dissimilatory iron reduction. This was a first step toward using geophysical data to predict behavior and kinetics of iron reduction more quantitatively. We determined that certain diagnostic reactions (reductive dissolution, acid-base reactions, and surface adsorption) control changes in electrical conductivity regardless of the reaction mechanism.

After completion of our batch experiments, we measured electrical conductivity in flow-through column experiments with ongoing iron reduction in collaboration with Flynn Picardal at Indiana University and Eric Roden of the University of Wisconsin. We set up a column that was filled with iron-bearing sediments and inoculated with an environmental culture, and fabricated and inserted electrodes in the column. Sterile PIPES-buffered artificial groundwater was amended with acetate to encourage iron reduction. Acetate consumption and the reduction of iron oxides observed in the column effluent did not correspond to changes in bulk electrical conductivity. Bulk electrical conductivity during Fe reduction was observed to increase more rapidly than predicted based upon in vivo flask experiments or measured fluid conductivity in the column: during the last two months of the experiment, bulk electrical conductivity increased by a factor of 3. [Fe(II)] did not show a similar trend. We suggested that sorbed $\mathrm{Fe}(\mathrm{II})$ and/or biofilms growing on mineral surfaces created a new conductive pathway. In addition to the diagnostic reactions above, we also found that biosynthesis likely needs to be considered in these systems.

To explore the important of sorbed Fe(II), we constructed additional column reactors, and found that an abiotic control showed no increase in conductivity due to $\mathrm{Fe}$ (II) adsorption, suggesting that biofilms were controlling the changes in electrical conductivity. We modeled this decoupling between fluid and bulk electrical conductivity with percolation theory and the addition of biofilms $33 \mathrm{x}$ more conductive than the pore fluid and occupying 3\% of the pore space, and found we could fit the laboratory data well.

Finally, we used PHREEQC to estimate the extent of biomass growth in the flowthrough column reactors. We coupled the output of this model with a pore-scale electrical model to estimate the electrical conductivity of the reactor given growing biomass. While the models are non-unique, they provide a starting point for future research focused on quantifying the electrical conductivity of biofilms.

Student training: This grant supported the research of one Ph.D. student, Aaron Regberg, as well as one undergraduate, Rowan Cockett. 


\section{Products delivered (*student author):}

1. Regberg, A.*, Singha K., Tien, M., Picardal, F., Zhang, Q., Schieber, J., Roden, E. and Brantley S. L. (2011). Electrical conductivity as an indicator of iron reduction rates in abiotic and biological systems. Water Resources Research, 47, W04509, doi:10.1029/2010WR009551, $14 \mathrm{p}$.

2. Regberg, A.*, Cockett, R.*, Pidlisecky, A., Brantley, S., and K. Singha. Modeling biofilm conductivity with reactive transport and pore-scale electrical conduction models. In final stages of preparation for submission to Chemical Geology.

3. Regberg, A.*, Singha, K., Bond, D. R., Zheng, Q., Picardal, F., Roden, E. E., and Brantley, S. (2010). Quantifying the Effect of Dissimilatory Iron Reduction on Bulk Electrical Properties: Does Iron Reduction Increase Electrical Conductivity? Annual American Chemical Society Meeting, March 21-25, 2010, San Francisco.

4. Singha, K., Regberg, A.*, Day-Lewis, F.D. (2009) Can electrical geophysics help quantify reaction rates in situ? Penn State Center of Environmental Kinetics Analysis Annual All-Hands Meeting. Oct 1, 2009, University Park, PA. (Invited.)

5. Regberg, A.*, Singha, K., Brantley, S. (2009). Electrical Conductivity Associated with Dissimilatory Iron Reduction: Are Microbial Biofilms Conductive? Goldschmidt, June 21-26, 2009, Davos, Switzerland.

6. Regberg, A.*, Zheng, Q., Picardal, F., Singha, K., Brantley, S. (2008). Anomalous electrical signals associated with microbial activity: results from iron- and nitratereducing columns. EOS Trans. AGU 89(53), Fall Meet. Suppl. Abstract H33G1115.

7. Regberg, A.*, Singha, K., Tien, M., and Brantley, S. (2008). The Link Between Electrical Fluid Conductivity and Microbial Iron Reduction. Center for Environmental Kinetics Annual All-Hands Meeting, Oct 22-24, 2008, University Park, PA.

8. Singha, K., Regberg, A.*, Brantley, S., and Tien, M. (2008). Moving toward quantifying kinetics in the field: Where we are and what we need. AGU Biogeophysics Chapman Conference, Oct 13-16, 2008, Portland, ME. (Invited).

9. Regberg, A.*, Singha, K., Brantley, S., and Tien, M. (2008). Interpreting changes in fluid conductivity due to the reductive dissolution of iron oxides. AGU Biogeophysics Chapman Conference, Oct 13-16, 2008, Portland, ME.

10. Regberg, A.*, Singha, K., Brantley, S., and Tien, M. (2008). Using Conductivity to Measure Iron Reduction Rates from Flask to Field. Geochimica et Cosmochimica Acta, 72, 12, A782; Goldschmidt Conference, July 13-18, 2008, Vancouver, Canada. 